\title{
Configuration mixing in nucleon-induced pre-equilibrium reactions
}

\author{
C.A. Soares Pompeia ${ }^{1}$, B.V. Carlson ${ }^{1, a}$, and F.B. Guimarães ${ }^{2}$ \\ ${ }^{1}$ Institute Tecnológico de Aeronáutica, 12228-900 São José dos Campos SP, Brazil \\ 2 Institute de Estudos Avançados - CTA, 12228-900 São José dos Campos SP, Brazil
}

\begin{abstract}
We present a unified semiclassical model of nucleon-induced pre-equilibrium reactions that permits the simulation of varying degrees of internal configuration mixing. We can reproduce the limits of no intraclass transitions, assumed in the hybrid Monte Carlo simulation (HMS) model, or of intraclass equilibrium, assumed in exciton models. We compare the two limiting cases with a natural model, in which the intraclass transitions are treated on an equal footing with the others. Monte Carlo simulations of the models with no intraclass transitions and with the natural ones yield very similar emission spectra, which are quite different from the exciton model ones. To reproduce standard exciton model spectra, the model simulations require an intraclass transition rate a thousand times larger than the natural one. Calculations using simple shell-model interactions show the average squared interclass and intraclass matrix elements to be similar in magnitude, as assumed in the natural model.
\end{abstract}

\section{Introduction}

Pre-equilibrium emission plays an ever more important role in nucleon-induced reactions as the incident energy increases above about $10 \mathrm{MeV}$. Models to describe such reactions were developed long ago. The first of these was the intranuclear cascade model, which follows sequences of two-body collisions through the spatial volume of the nucleus $[1,2]$. A different approach was introduced by Griffin, who described a pre-equilibrium reaction in terms of a sequence of collisions that populate increasingly complex configurations of single-particle-excitations, thus transferring the cascade from geometrical space to energy space [6]. The two best-known semiclassical pre-equilibrium models, the hybrid [7-9] and exciton models [10-13], are based on such an approach. Although more sophisticated quantum mechanical models have been developed over the years [14-21], many calculations still rely on these older, but very successful, semiclassical models [22-24].

The differences between the hybrid and exciton models were a point of contention for many years. These were clarified in an analysis by Bisplinghoff [25], who found the fundamental difference between the two to be their assumptions concerning the degree of mixing among configurations with the same number of particles and holes. The exciton model assumes the mixing to be complete, so that equilibrium among these configurations is reached, while the hybrid model assumes that no configuration mixing occurs at all.

Later, Blann proposed an alternative to the hybrid model, called the hybrid Monte Carlo simulation (HMS) model $[26,27]$, which uses only those lowest-order exciton densities that are consistent with the kinematics of nucleon-nucleon scattering in a nucleus [9]. The HMS model contains no configuration mixing and presents a conceptuallyconsistent alternative to the exciton model.

${ }^{a}$ Presenting author, e-mail: brett@ita.br

\section{A unified semiclassical pre-equlibrium model}

Semi-classical pre-equilibrium models are usually formulated on the basis of a set of independent single-particle states of the composite nucleon-nucleus system. In the ground state of the system, each single-particle state below the Fermi energy is occupied by a single nucleon. In an excited state, one or more of the particles occupy states above the Fermi energy, leaving the same number of holes below the Fermi energy. Each distinct arrangement of the particles and holes (taking into account indistinguishability and the exclusion principle) defines a particle-hole configuration. Energy is assumed to be conserved at all times, so that only the subset of the particlehole configurations with the same excitation energy, $E^{*}$, needs to be considered in a given reaction. The exciton number of a configuration is defined as the sum of the numbers of particles and holes. An exciton class is defined as the set of configurations with the same numbers of particles and of holes and, thus, the same exciton number. In the exciton model, it is common practice to suppress one of the holes, which the model assumes to be fixed at the Fermi energy, when labeling configurations. We will not do so here.

We assume a nucleon-induced reaction to be initiated by the fusion of the projectile nucleon and the target to form a $2 \mathrm{p}-2 \mathrm{~h}$ configuration in which one hole is initially at the Fermi surface. Due to the two-body nature assumed for the internal nuclear transitions, these can excite another particlehole pair and increase the exciton number by two, scatter two particles, two holes or a particle and a hole and leave the exciton number the same or annihilate a particle-hole pair and reduce the exciton number by two. The transitions that do not alter the exciton number mix the configurations within each exciton class. These enter neither in the HMS nor in the exciton models, although for very different reasons.

We take the single-particle spectrum of the model to be determined so that the most deeply-bound nucleon of the A+1 target-nucleon system is bound by $45 \mathrm{MeV}$ while the projectile nucleon, if it were in its fundamental state at the Fermi energy, would be bound by a separation energy 
$B$ of about $8 \mathrm{MeV}$. The initial configuration of the system is thus a 1p-1h one, with the hole at the Fermi energy and the particle (the projectile nucleon) occupying the single-particle state closest in excitation energy $E^{*}$ to $E_{n}+B \mathrm{MeV}$, where $E_{n}$ is the incident center-of-mass energy. We do not distinguish between neutrons and protons.

We treat each particle-hole configuration explicitly. Thus, we consider the $1 \mathrm{p}-1 \mathrm{~h}$ configuration consisting of a particle of energy $\varepsilon_{p 1}$ and a hole of energy $\varepsilon_{h 1}$ to be distinct from the $1 \mathrm{p}-1 \mathrm{~h}$ configuration consisting of a particle of energy $\varepsilon_{p 2}$ and a hole of energy $\varepsilon_{h 2}$ if $p 1 \neq p 2$ or $h 1 \neq h 2$. We label each particle-hole configuration by a letter from the beginning of the alphabet $a, b, c \ldots$ as well as a class label $l, m, n \ldots$ denoting the exciton number, that is, the total number of particles and holes.

Master equation. The configuration occupation probabilities are governed by a master equation,

$$
\hbar \frac{d P_{n a}}{d t}=\sum_{m b} \Lambda_{n a, m b} P_{m b}-\Gamma_{n a} P_{n a},
$$

where the total decay width of the configuration $n a$ is given in terms of the partial transition widths $\Lambda_{l c, n a}$ and partial emission widths $\Gamma_{e, n a}$ by

$$
\Gamma_{n a}=\sum_{l c} \Lambda_{l c, n a}+\sum_{e} \Gamma_{e, n a} .
$$

Emission. We estimate emission using the usual Weisskopf expression. We take the partial width for nucleon emission from the configuration $n a$ in an interval $\Delta E$ of emission energy $e$ to be

$$
\Gamma_{e, n a}=\frac{g_{s} \mu}{\pi^{2} \hbar^{2}} e \sigma_{a b s}(e) \Delta E,
$$

if the configuration contains a particle of energy $e+B$, where $B$ is the separation energy, and as zero otherwise. The total emission width of a configuration is the sum of the partial widths of each of the particles that can be emitted.

Transitions. We consider transitions induced by energyconserving two-body collisions and denote the partial width for the transition from $m b$ to $n a$ as $\Lambda_{n a, m b}$. We assume microscopic reversibility, so that $\Lambda_{n a, m b}=\Lambda_{m b, n a}$. The nonzero partial transition widths increase the exciton number by 2 , $\Lambda_{n+2 a, n b}$, leave it the same, $\Lambda_{n a, n b}$, or decrease it by $2, \Lambda_{n-2 a, n b}$. The two-body collision inducing the transition from a configuration $m b$ to a configuration $n a$ is unique. We associate a single value to all non-zero partial transition widths. We take this to be the value associated with the average squared matrix element of the exciton model, which we take to be,

$$
\Lambda_{n a, m b}=\left\{\begin{aligned}
2 \pi \frac{f_{0}}{A^{3} E^{*}} & f_{0} \approx 1350 \mathrm{MeV}, \text { when } m b \rightarrow n a \\
0 & \text { otherwise }
\end{aligned}\right.
$$

where $f_{0}$ was adjusted so as to best reproduce the expression given in equation (29) of ref. [24]. We call this the natural model.

The HMS and exciton model limits. By varying the transition rates among the different classes of states, we can study the effects of the different hypotheses of the two models. By taking $\Lambda_{n a, n b} \rightarrow 0$, we eliminate the internal mixing in each exciton class. The lack of internal mixing is the basic distinguishing characteristic of the hybrid and HMS models.
Within the unified model, this no-mixing limit furnishes a model similar to the HMS one.

When the intraclass partial widths are much larger than the interclass ones,

$$
\Lambda_{n a, n b}>>\Lambda_{n a, m b}, \Lambda_{m b, n a} \quad m=n \pm 2,
$$

we expect the configurations within an exciton class to reach equilibrium before the occurence of transitions between classes. In this limit, the model reduces to the usual exciton model, in which system evolution and emission rates depend only on the populations of the exciton classes and not on those of the individual configurations. The transitions within a class then no longer contributes to the evolution of the system.

More details of the unified model can be found in ref. [28].

\section{Numerical results}

The number of particle-hole configurations in a typical unified-model calculation is usually extremely large, so that direct solution of the master equation is simply not viable. We use instead a Monte Carlo simulation method to obtain emission spectra.

We denote as natural model simulations those in which the partial widths for internal transitions are identical and by no-mixing simulations those in which internal mixing has been eliminated by taking $\Lambda_{n a, n b} \rightarrow 0$. We denote as complete-mixing simulations those in which the transitions between configurations of the same exciton class have been taken to be a factor of 1000 larger than those used in the natural model. We also perform standard exciton model calculations using the class transition and emission rates obtained from the sums over configurations.

In the simulations, each Monte Carlo cascade was initiated from a $1 \mathrm{p}-1 \mathrm{~h}$ configuration in which the excitation energy is carried by the particle and the hole is at the Fermi surface. This hole is normally maintained fixed at the Fermi surface when pre-equilibrium exciton densities and transition rates are calculated. We perform complete-mixing and standard exciton model calculations with the hole fixed and with it free. We denote the fixed-hole calculations as Mix1 and the calculations in which the hole is free to interact as Mix0. In the natural and no-mixing simulations, the hole is free to interact at all stages of the reaction.

In figure 1, we show spectra of the first emitted nucleon from a nucleon +56 -nucleon system at an incident centerof-mass-energy of $50 \mathrm{MeV}$ at several stages of the reaction. The Monte Carlo simulations are shown as histograms while standard exciton model calculations are displayed as thin lines. The spectra labeled $2 p$ correspond to emission from the $2 \mathrm{p}-2 \mathrm{~h}$ stage of the reaction before the $3 \mathrm{p}-3 \mathrm{~h}$ stage is reached. The spectra corresponding to emission of the first nucleon from any stage of the composite system are also shown. We note the excellent agreement between the complete-mixing and standard exciton model spectra, in both the fixed-hole and free-hole cases. The no-mixing and natural model simulations are also in very close agreement with one another. The fixed-hole spectra are substantially harder than the no-mixing and natural model ones at each of the initial stages and in the overall one-nucleon emission spectrum, while the free-hole spectra are much softer. 


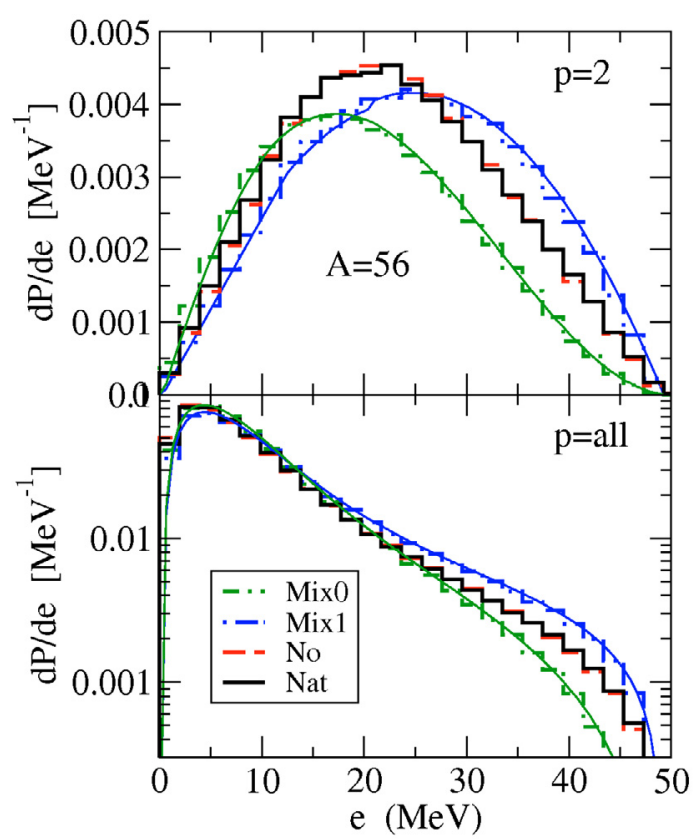

Fig. 1. Spectra of the first nucleon emitted from the $2 p-2 h$ and all stages of a nucleon +56 -nucleon system at an incident energy of $50 \mathrm{MeV}$. The Monte Carlo calculations are represented by histograms as labeled in the figure and discussed in the text. The thin lines correspond to standard exciton model calculations.

One might ask if the factor of 1000 multiplying intraclass partial widths is necessary to provide the equilibrium of the complete-mixing model. We reply with figure 2 , where we show spectra of the first emitted nucleon from the nucleon + 56-nucleon system at an incident center-of-mass-energy of $50 \mathrm{MeV}$ at the $2 \mathrm{p}-2 \mathrm{~h}$ and at all stages of the reaction for values of the factor multiplying intraclass transitions of 10 , 100 and 1000. Recall that the natural model uses a factor of 1 , but does not fix the hole at the Fermi energy. The fixed-hole simulations using an intraclass factor of 10 fall closer to the natural model spectra than to the standard exciton model one (thin line). The simulations using a factor of 100 come closer, but are still very distinguishable from the standard exciton model calculations, which lie atop the simulations using a factor of 1000. The free-hole simulations (not shown) fall in an almost symmetrical fashion on the other side of the natural model calculations, tending toward the corresponding standard exciton model calculation as the multiplicative factor tends to 1000 . We conclude that a factor of the order of 1000 is indeed necessary to provide class equilibrium in both complete-mixing simulations.

One might then ask if the intraclass partial widths are not in fact 1000 times larger than the interclass ones. The average partial widths can be estimated microscopically within the shell model as the ratio of the sum of the squares of matrix elements of the two-body interaction coupling classes of configurations and the corresponding densities of available states. In much the same manner that calculation of the density of available states can be reduced to a convolution over simpler densities of states [29], the calculation of summed squared matrix elements can be reduced to convolutions over densities

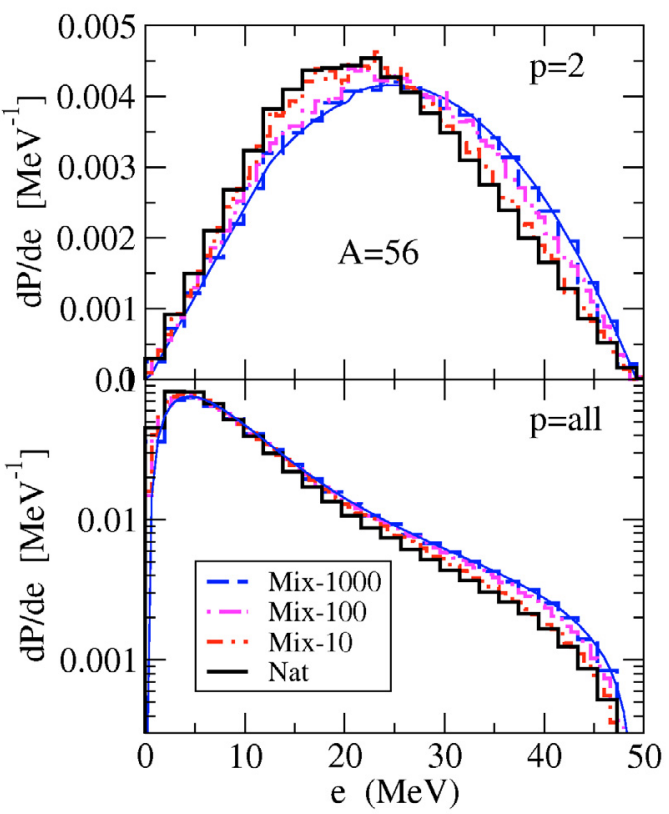

Fig. 2. Spectra of the first nucleon emitted from the $2 \mathrm{p}-2 \mathrm{~h}$ and all stages of a nucleon +56 -nucleon system at an incident energy of $50 \mathrm{MeV}$ for various values of the intraclass transition strength. The Monte Carlo calculations are represented by histograms as labeled in the figure and discussed in the text. The thin lines correspond to standard exciton model calculations.

and summed squared two-body matrix elements that either increase (or decrease) the exciton number by two or leave it unchanged [30]. Typical values of these averaged two-body matrix elements are shown in figure 3 . We note that the partial widths for transitions induced by holes are about an order of magnitude larger than the partial widths of those induced by particles, in both the interclass and intraclass cases. The transitions induced by a particle and a hole, possible in the intraclass case, have partial widths which lie between the particle-induced and hole-induced ones. However, the densities of available states for hole-induced transitions are much smaller than those for particle-induced ones, as evidenced in the figure by the average values, which lie close to the particle ones. Comparing the average values in the upper and lower panels, we conclude that the intraclass and interclass partial widths are in fact about equal, as assumed in the natural model.

\section{Conclusions}

We have developed a unified model of semi-classical nucleoninduced pre-equilibrium reactions and used it to simulate the very different hypotheses of no mixing and of complete mixing, as well as an intermediate case that we call the natural model. We have seen that the no-mixing and natural model emission spectra are almost identical. This tells us that the internal configuration mixing of the natural model is little more effective than no mixing at all. Second, we have seen that the complete-mixing results are identical to the standard exciton model results but are quite different from the no-mixing and natural ones. This tells us two things: that the complete-mixing 


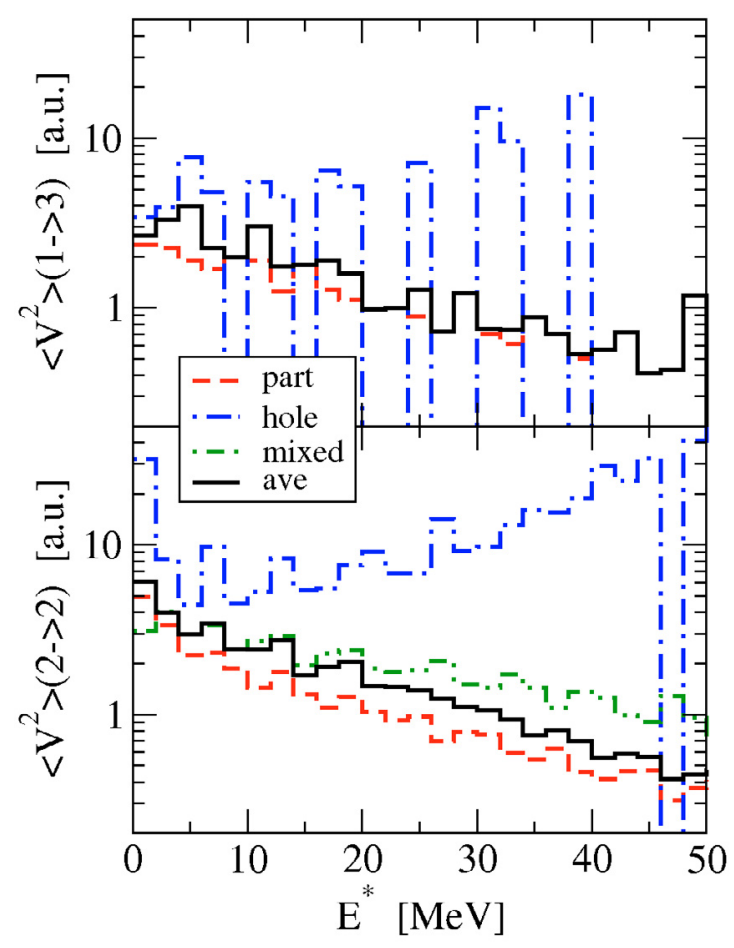

Fig. 3. Average squared two-body matrix elements for interclass (upper panel) and intraclass (lower panel) transitions as a function of the excitation energy.

simulations with transitions a thousand times stronger than the natural ones do indeed attain the class equilibrium presumed in the standard exciton model and that the no-mixing and natural model simulations thus describe emission from systems that are far from this equilibrium. The fixed-hole complete-mixing model also suffers from the conceptual inconsistency of fixing a hole at the Fermi surface while allowing all other degrees of freedom to interact strongly. We conclude that the no-mixing and natural models provide the more consistent description of pre-equilibrium reactions and that the intraclass equilibrium of the complete-mixing and exciton models is not attained in the early stages of these reactions.

The results obtained here are also pertinent to quantummechanical models of pre-equilibrium reactions. Our conclusions tell us that the use of residual-system statistics based on configuration mixing, such as those used in most multistep compound models $[14,15,17]$ and in the multistep direct model of Tamura, Udagawa and Lenske [16], cannot be justified, at least not in the early stages of a reaction.
The authors thank O.F. de Lima, A. Passaro and R.Y. Tanaka, of the Instituto de Estudos Avançados - CTA, for help in the preparation and use of a parallel version of the computer code on the BELIEVEe II cluster. C.A.S. Pompeia acknowledges the support of CAPES. B.V. Carlson acknowledges partial support from FAPESP and the CNPq.

\section{References}

1. M.L. Goldberger, Phys. Rev. 74, 1268 (1948).

2. N. Metropolis, R. Bivins, M. Storm, A. Turkevich, J.M. Miller, G. Friedlander, Phys. Rev. 110, 185 (1958); 110, 204 (1958).

3. H.W. Bertini, Phys. Rev. 131, 1801 (1963).

4. Y. Yariv, Z. Fraenkel, Phys. Rev. C 20, 2227 (1979); C 24, 488 (1981).

5. J. Cugnon, Nucl. Phys. A 462, 751 (1987); J. Cugnon, C. Volant, S. Vuillier, Nucl. Phys. A 620, 475 (1997).

6. J.J. Griffin, Phys. Rev. Lett. 17, 478 (1966); Phys. Lett. B 24, 5 (1967).

7. M. Blann, Phys. Rev. Lett. 27, 337 (1971).

8. M. Blann, Phys. Rev. Lett. 28, 757 (1972).

9. M. Blann, H.K. Vonach, Phys. Rev. C 28, 1475 (1983).

10. C.K. Cline, M. Blann, Nucl. Phys. A 172, 225 (1971).

11. C.K. Cline, Nucl. Phys. A 210, 590 (1973).

12. J. Dobes, E. Beták, Nucl. Phys. A 272, 353 (1976).

13. E. Gadioli, E. Gadioli-Erba, Nucl. Instrum. Meth. 146, 265 (1977).

14. D. Agassi, H.A. Weidenmüller, G. Mantzouranis, Phys. Rep. 22, 145 (1975).

15. H. Feshbach, A. Kerman, S. Koonin, Ann. Phys. (NY) 125, 429 (1980).

16. T. Tamura, T. Udagawa, H. Lenske, Phys. Rev. C 26, 379 (1982).

17. H. Nishioka, J.J.M. Verbaarschot, H.A. Weidenmüller, S. Yoshida, Ann. Phys. (NY) 172, 67 (1986).

18. H. Nishioka, H.A. Weidenmüller, S. Yoshida, Ann. Phys. (NY) 183, 166 (1988).

19. J.M. Akkermans, A.J. Koning, Phys. Lett. B 234, 417 (1990).

20. A.J. Koning, J.M. Akkermans, Ann. Phys. (NY) 208, 216 (1991).

21. A.J. Koning, M.B. Chadwick, Phys. Rev. C 56, 970 (1997).

22. M. Blann, Ann. Rev. Nucl. Sci. 25, 123 (1975).

23. C. Kalbach, J. Phys. G. 21, 1519 (1995).

24. A.J. Koning, M.C. Duijvestijn, Nucl. Phys. A 744, 15 (2004).

25. J. Bisplinghoff, Phys. Rev. C 33, 1569 (1986).

26. M. Blann, Phys. Rev. C 54, 1341 (1996).

27. M. Blann, M.B. Chadwick, Phys. Rev. C 57, 233 (1998).

28. C.A. Soares Pompeia, B.V. Carlson, Phys. Rev. C 74, 054609 (2006).

29. C.K. Cline, Nucl. Phys. A 195, 353 (1972).

30. B.V. Carlson, F.B. Guimarães (in preparation). 